NEW ZEALAND JOURNAL OF MATHEMATICS

Volume 52 (2022), 821-826

https://doi.org/10.53733/205

\title{
ON THE HOMEOMORPHISM PROBLEM FOR 4-MANIFOLDS
}

\author{
CAmeron McA. Gordon \\ (Received 28 January, 2022)
}

\begin{abstract}
We show that there is no algorithm to decide whether or not a given 4-manifold is homeomorphic to the connected sum of 12 copies of $S^{2} \times S^{2}$.
\end{abstract}

This paper is dedicated to the memory of Vaughan Jones. Vaughan had a huge impact on mathematics, and on the community of mathematicians. His New Zealand summer conferences, some of which I had the good fortune to attend, were legendary. Breakfast, talks until lunch, afternoons free to indulge in the activity of your choice (of course for Vaughan it was windsurfing), dinner, followed by another talk, enlivened by the prandial and postprandial wine. Then there was the famous Knots in Hellas '98 Conference in Delphi, where (among other things) Vaughan was presented with the keys to the city by the Mayor of Delphi, and where one evening his Fields Medal went missing (but luckily showed up again the next morning). I also enjoyed our many games of squash over the years, in many different locations. We will miss Vaughan, and will remember him with fondness and respect.

\section{Introduction}

In [Mar] Markov showed that the homeomorphism problem for closed 4-manifolds is algorithmically unsolvable. In fact he showed that for some integer $k$ the recognition problem for $\#_{k}\left(S^{2} \times S^{2}\right)$, the connected sum of $k$ copies of $S^{2} \times S^{2}$, is unsolvable, i.e. there is no algorithm to decide whether or not a given 4 -manifold is homeomorphic to $\#_{k}\left(S^{2} \times S^{2}\right)$.

To describe this in more detail, let us define a $k$-relator Adjan-Rabin set to be a recursively enumerable set $\mathcal{P}$ of finite $k$-relator group presentations, such that there is no algorithm to decide whether or not the group presented by a given $P \in \mathcal{P}$ is trivial. Such sets were shown to exist, for some $k$, by Adjan [A] and Rabin [Ra], using the existence, proved by Novikov [N] and Boone [Boo], of a finitely presented group with unsolvable word problem. Markov showed that if there exists a $k$-relator Adjan-Rabin set then the recognition problem for $\#_{k} S^{2} \times S^{2}$ is unsolvable.

The author showed $[\mathrm{G}]$ that from a finite $m$-relator presentation of a group with unsolvable word problem one can construct an $(m+2)$-relator Adjan-Rabin set; there is also an account of this work in the survey article [Mi]. In [Bor] Borisov constructed a finite 12-relator presentation of a group with unsolvable word problem. It follows that the recognition problem for $\#_{14}\left(S^{2} \times S^{2}\right)$ is unsolvable. See $[\mathrm{S} 1],[\mathrm{S} 2],[\mathrm{CL}]$.

The purpose of the present note is to offer the following improvement.

Theorem 1.1. The recognition problem for $\#_{12}\left(S^{2} \times S^{2}\right)$ is unsolvable.

A natural question is whether $k$ can be reduced further, in particular whether it can be reduced to 0 . 
Question 1.2. Is the recognition problem for $S^{4}$ unsolvable?

The recognition problem for $S^{n}$ is unsolvable for $n \geq 5$ [VKF, Appendix by S.P. Novikov], and solvable for $n \leq 3[\mathrm{Ru}],[\mathrm{T}]$.

The proof of Theorem 1.1 has two parts, one algebraic and the other topological, each enabling $k$ to be reduced by 1 . The first is discussed in Section 2 , and the second in Section 3.

\section{The Algebra}

Let $\left(x_{1}, \ldots, x_{n}: r_{1}, \ldots, r_{m}\right)$ be a finite presentation of a group $G$. Let $\bar{x}_{i}$ denote the image of $x_{i}$ in $G /[G, G]$.

Consider the following property:

(2.1) there exists $p, 1 \leq p \leq n$, such that for $1 \leq i \leq p, \bar{x}_{i}$ has finite order $q_{i} \geq 1$, where $\operatorname{gcd}\left(q_{1}, \ldots, q_{p}\right)=1$.

Lemma 2.1. If there exists a group with unsolvable word problem having a finite $m$ relator presentation that satisfies (2.1), then there exists an $(m+1)$-relator AdjanRabin set.

Proof. We modify the construction given in $[\mathrm{G}]$. Let $\left(x_{1}, \ldots, x_{n}: r_{1}, \ldots, r_{m}\right)$ be a presentation of a group $G$ with unsolvable word problem that satisfies (2.1). By taking a minimal set $\left\{x_{1}, \ldots, x_{p}\right\}$ with property (2.1) we may assume that the $q_{i}$ are all distinct. Let $q=\max \left\{q_{1}, \ldots, q_{p}\right\}$.

Let $W\left(x_{1}, \ldots, x_{n}\right)$ denote the set of words in $\left\{x_{1}, \ldots, x_{n}\right\}$, i.e. the set of expressions of the form $x_{i_{1}}^{\epsilon_{1}} \ldots x_{i_{r}}^{\epsilon_{r}}, x_{i_{j}} \in\left\{x_{1}, \ldots, x_{n}\right\}, \epsilon_{j}= \pm 1$. For $w \in W\left(x_{1}, \ldots, x_{n}\right\}$, let $Q_{w}$ be the presentation with generators $x_{1}, \ldots, x_{n}, a, \alpha, b, \beta$, and relators $r_{1}, \ldots, r_{m}$ together with

(i) $a \alpha a^{-1}=b^{2}$

(ii) $\alpha a \alpha^{-1}=b \beta b^{-1}$

(iii) $a^{-q_{i}} x_{i} \alpha^{q_{i}}=\beta^{-i} b \beta^{i}, \quad 1 \leq i \leq p$

(iv) $a^{-(q+i)} x_{i} \alpha^{(q+i)}=\beta^{-i} b \beta^{i}, \quad p+1 \leq i \leq n$

(v) $\left[w, \alpha^{2}\right]=\beta^{-(n+1)} b \beta^{(n+1)}$

where $[x, y]$ means $x y x^{-1} y^{-1}$.

Let $G_{w}$ be the group presented by $Q_{w}$. We can apply the following Tietze transformations to $Q_{w}$. Using (i), express $\alpha$ in terms of $a$ and $b$, substitute this expression for the occurrences of $\alpha$ in the other relations, then delete $\alpha$ from the generators and (i) from the relations. Now from (ii) express $\beta$ in terms of $a$ and $b$, substitute for $\beta$ into the other relations, and delete $\beta$ and relation (ii). Using relations (iii) and (iv) we can now write the $x_{i}$ as words in $a$ and $b$, substitute these into the relators $r_{j}$, getting relators $r_{j}^{\prime}$ that are words in $a$ and $b$, substitute for the $x_{i}$ in $w$ in (v), and finally delete the $x_{i}$ and relations (iii) and (iv).

We are left with a presentation $P_{w}$ of $G_{w}$ with two generators, $a$ and $b$, and $(m+1)$ relations: the relators $r_{j}^{\prime}, 1 \leq j \leq m$, and the transformed relation (v). We claim that $\left\{P_{w}: w \in W\left(x_{1}, \ldots, x_{n}\right)\right\}$ is an Adjan-Rabin set.

Let $U$ denote the set of elements listed on the right-hand side of the relations (i) - (v). By examining the possible cancellation in $u_{1}^{\epsilon_{1}} u_{2}^{\epsilon_{2}}$, where $u_{1}$ and $u_{2}$ are distinct elements of $U$ and $\epsilon_{i}= \pm 1, i=1,2$, it is easy to see that a non-empty reduced word in the elements of $U$ has positive length when expressed as a reduced word in $b$ and $\beta$. Thus $U$ is a basis for a free subgroup of the free group $F(b, \beta)$. 
Similarly, if $[w] \neq 1$ in $G$, one sees that the set of elements on the left-hand side of the relations is a basis for a free subgroup of the free product $G * F(a, \alpha)$. Hence if $[w] \neq 1$ in $G$ then $G_{w}$ is a free product with amalgamation $(G * F(a, \alpha)) *_{F} F(b, \beta)$, where $F$ is free of rank $(n+3)$. In particular $G_{w} \neq 1$.

If $[w]=1$ in $G$ then (v), together with the relators $r_{1}^{\prime}, \ldots, r_{m}^{\prime}$, implies that $b=1$, and therefore $G_{w}$ is cyclic, generated by $a$. Also, $\alpha=1$ by (i). Relations (iii) give $x_{i}=a^{q_{i}}, 1 \leq i \leq p$. By condition (2.1) $x_{i}$ maps to an element in $G_{w}$ of order dividing $q_{i}$; hence in $G_{w}$ we have the relations $a^{q_{i}^{2}}, 1 \leq i \leq p$. Since $\operatorname{gcd}\left(q_{1}, \ldots, q_{p}\right)=1$, this implies $a=1$, and hence $G_{w}=1$.

Thus $G_{w}=1$ if and only if $[w]=1$ in $G$. Since $G$ has unsolvable word problem, $\left\{P_{w}: w \in W\left(x_{1}, \ldots, x_{n}\right)\right\}$ is an Adjan-Rabin set.

Theorem 2.2. There exists a 13-relator Adjan-Rabin set.

Proof. Matijasevič [Mat] has shown that there exists a semigroup $S$ having a presentation with two generators and three relations, and a positive word $W_{0}$ in the generators, such that there is no algorithm to decide, for an arbitrary positive word $W$ in the generators, whether or not $W$ and $W_{0}$ represent the same element of $S$. Borisov shows that this may be used to construct a presentation, with generators $a, b, c, d$, and $e$ and 12 relations, of a group $\Gamma^{\prime}$ with unsolvable word problem; see [Bor, §3]. Among the relations are

$$
\mu_{i} d \mu_{i}^{-1}=d^{\alpha}, \mu_{i}^{-1} e \mu_{i}=e^{\alpha}, \quad i=1,2
$$

where $\mu_{1}$ and $\mu_{2}$ are words in $a$ and $b$ and $\alpha$ is an arbitrary integer $>3$. However, an examination of the proof in [Bor] that $\Gamma^{\prime}$ has unsolvable word problem shows that these relations may be replaced by

$$
\mu_{i} d \mu_{i}^{-1}=d^{u}, \mu_{i}^{-1} e \mu_{i}=e^{v}, \quad i=1,2
$$

for any integers $u, v>3$.

So, taking $u=4, v=5$, we get a 12-relator presentation of a group with unsolvable word problem where the generators $d$ and $e$ have the property that the order of $\bar{d}$ divides 3 and the order of $\bar{e}$ divides 4 . The result now follows from Lemma 2.1.

\section{The Topology}

We briefly summarize Markov's argument [Mar]. For other discussions see [S1], $[\mathrm{CL}],[\mathrm{K}]$. We will not discuss the algorithmic aspects of the PL constructions involved; these are dealt with in [BHP]; see also [S1].

Let $P=\left(x_{1}, \ldots, x_{n}: r_{i}, \ldots, r_{k}\right)$ be a finite presentation of a group $G_{P}$.

Attach $n$ 1-handles to $B^{5}$ so as to get an orientable 5 -manifold $V$ with $\pi_{1}(V) \cong$ $F\left(x_{i}, \ldots, x_{n}\right)$. Let $\gamma_{1}, \ldots, \gamma_{k}$ be disjoint circles in $\partial V$ such that $\left[\gamma_{j}\right]$ is conjugate to $r_{j}$ in $\pi_{1}(V) \cong \pi_{1}(\partial V), 1 \leq j \leq k$. Since homotopy implies isotopy for 1-manifolds in a 4-manifold by general position, $\gamma=\bigcup_{j=1}^{k} \gamma_{j}$ is well-defined up to isotopy in $\partial V$. Let $N_{P}$ be obtained by attaching 2-handles $H\left(\gamma_{j}\right)$ to $V$ along $\gamma_{j}, 1 \leq j \leq k$. We express this as $N_{P}=V \cup H(\gamma)$. Clearly $\pi_{1}\left(N_{P}\right) \cong G_{P}$. Also, since $N_{P}$ has a 2-dimensional spine, a general position argument shows that inclusion $\partial N_{P} \rightarrow N_{P}$ induces an isomorphism on fundamental groups. 
The homeomorphism type of $N_{P}$ depends only on $P$ and a choice of framing of the normal bundle of $\gamma_{j}$ in $\partial V, 1 \leq j \leq k$. The set of such framings is a $\mathbb{Z}_{2}$-torsor. To ensure that $N_{P}$ depends only on $P$ we note that there is an obvious embedding of $V$ in $\mathbb{R}^{5}$. Then any circle in $\partial V$ bounds a disk in $\mathbb{R}^{5}$, and in attaching a 2-handle along such a circle we will always choose the framing that extends over the normal bundle of the disk, and call this the 0-framing.

Let $\alpha_{1}, \ldots, \alpha_{n}$ be disjoint circles in a 4-ball in $\partial N_{P} \cap \partial V$, and let $W_{P}$ be the result of attaching 2-handles $H\left(\alpha_{i}\right)$ to $N_{P}$ along $\alpha_{i}, 1 \leq i \leq n$ (with the 0 -framing). Let $M_{P}=\partial W_{P}$. Then $\pi_{1}\left(M_{P}\right) \cong \pi_{1}\left(W_{P}\right) \cong \pi_{1}\left(N_{P}\right) \cong G_{P}$.

Markov's key observation is the following.

Lemma 3.1. (Markov) $G_{P}=1$ if and only if $M_{P} \cong \#_{k}\left(S^{2} \times S^{2}\right)$.

Proof. Since $\pi_{1}\left(M_{P}\right) \cong G_{P}$ the "if" direction is clear.

For the converse, suppose $G_{P}=1$. Let $\beta_{1}, \ldots, \beta_{n}$ be disjoint circles in $\partial V$ that are dual to the co-cores of the 1-handles and disjoint from $\gamma$. Then we may regard $\beta_{1}, \ldots, \beta_{n}$ as lying in $\partial N_{P}$. Recalling that $\pi_{1}\left(\partial N_{P}\right) \cong G_{P}=1, \boldsymbol{\alpha}=\bigcup_{i=1}^{n} \alpha_{i}$ is isotopic to $\boldsymbol{\beta}=\bigcup_{i=1}^{n} \beta_{i}$ in $\partial N_{P}$. Therefore

$$
\begin{aligned}
W_{P} & =N_{P} \cup H(\boldsymbol{\alpha}) \\
& \cong N_{P} \cup H(\boldsymbol{\beta}) \\
& =(V \cup H(\boldsymbol{\gamma})) \cup H(\boldsymbol{\beta}) \\
& =(V \cup H(\boldsymbol{\beta})) \cup H(\boldsymbol{\gamma}) \\
& \cong B^{5} \cup H(\boldsymbol{\gamma}) \\
& \cong \natural_{k}\left(S^{2} \times D^{3}\right)
\end{aligned}
$$

where $\natural$ denotes boundary connected sum.

Hence $M_{P}=\partial W_{P} \cong \#_{k}\left(S^{2} \times S^{2}\right)$.

The above construction gives an algorithm that takes a finite $k$-relator presentation $P$ of a group $G_{P}$ and produces a closed 4 -manifold $M_{P}$ such that $G_{P}=1$ if and only if $M_{P} \cong \#_{k}\left(S^{2} \times S^{2}\right)$. To complete the proof of Markov's theorem we note that if $\mathcal{P}$ is a $k$-relator Adjan-Rabin set then an algorithm to decide, for a given $P \in \mathcal{P}$, whether or not the manifold $M_{P}$ is homeomorphic to $\#_{k}\left(S^{2} \times S^{2}\right)$ would give an algorithm to decide whether or not $G_{P}=1$, a contradiction.

We now describe a modification of the proof of Lemma 3.1 that enables us to replace $\#_{k}\left(S^{2} \times S^{2}\right)$ by $\#_{(k-1)}\left(S^{2} \times S^{2}\right)$.

Let $\boldsymbol{\alpha}^{\prime}=\bigcup_{i=1}^{n-1} \alpha_{i}, \boldsymbol{\beta}^{\prime}=\bigcup_{i=1}^{n-1} \beta_{i}$, define $W_{P}^{\prime}=N_{P} \cup H\left(\boldsymbol{\alpha}^{\prime}\right)$, and let $M_{P}^{\prime}=\partial W_{P}^{\prime}$. Note that $\pi_{1}\left(M_{P}^{\prime}\right) \cong \pi_{1}\left(W_{P}^{\prime}\right) \cong \pi_{1}\left(N_{P}\right) \cong G_{P}$.

Lemma 3.2. $G_{P}=1$ if and only if $M_{P}^{\prime} \cong \#_{(k-1)}\left(S^{2} \times S^{2}\right)$.

Proof. As before, the "if" direction is clear.

Assume $G_{P}=1$. Then, since $\pi_{1}\left(\partial N_{P}\right)=1 \boldsymbol{\alpha}^{\prime}$ is isotopic to $\boldsymbol{\beta}^{\prime}$ in $\partial N_{P}$, and as in the proof of Lemma 3.1

$$
W_{P}^{\prime} \cong\left(V \cup H\left(\boldsymbol{\beta}^{\prime}\right)\right) \cup H(\boldsymbol{\gamma})
$$

which is homeomorphic to $\left(S^{1} \times D^{4}\right) \cup H(\gamma)$. 
Let $a_{j}=\left[\gamma_{j}\right] \in \pi_{1}\left(S^{1} \times D^{4}\right) \cong \mathbb{Z}, 1 \leq j \leq k$. Orient $\gamma_{j}$ so that $a_{j} \geq 0$. Since $\pi_{1}\left(W_{P}^{\prime}\right)=1, \operatorname{gcd}\left(a_{1}, \ldots, a_{k}\right)=1$. Therefore, by a sequence of moves of the form

$$
\begin{aligned}
& a_{r} \mapsto a_{r}-a_{s} \\
& a_{j} \mapsto a_{j}, j \neq r,
\end{aligned}
$$

for some $r$ and some $s \neq r$ with $a_{s} \leq a_{r}$, followed by a permutation, we can transform $\left(a_{1}, \ldots, a_{k}\right)$ to $(1,0, \ldots, 0)$.

Since the above move can be realized by sliding $H\left(\gamma_{r}\right)$ over $H\left(\gamma_{s}\right)$,

$$
W_{P}^{\prime} \cong\left(S^{1} \times D^{4}\right) \cup H\left(\gamma^{\prime}\right)
$$

where $\left(\left[\gamma_{1}^{\prime}\right], \ldots,\left[\gamma_{k}^{\prime}\right]\right)=(1,0, \ldots, 0)$. Thus $W_{P}^{\prime}$ is homeomorphic to $B^{5}$ with $(k-1) 2$ handles attached with the 0 -framing, i.e. $\natural_{(k-1)}\left(S^{2} \times D^{3}\right)$. Hence $M_{P}^{\prime} \cong \#_{(k-1)}\left(S^{2} \times\right.$ $\left.S^{2}\right)$.

Proof of Theorem 1.1. This follows from Theorem 2.2 and Lemma 3.2.

\section{References}

[A] S.I. Adjan, Algorithmic unsolvability of problems of recognition of certain properties of groups, Dokl. Akad. Nauk SSSR 103 (1955), 533-535.

[Boo] W.W. Boone, The word problem, Ann. of Math. 70 (1959), 207-265.

[BHP $]$ W.W. Boone, W. Haken and V. Poenaru, On recursively unsolvable problems in topology and their classification, Contributions to Math. Logic (Colloquium, Hannover, 1966), pp. 37-74, North-Holland, Amsterdam, 1968.

[Bor] V.V. Borisov, Simple examples of groups with unsolvable word problem, Mat. Zametki 6 (1969), 521-532.

[CL] A.V. Chernavsky and V.P. Leksine, Unrecognizability of manifolds, Ann. Pure Appl. Logic 141 (2006), 325-335.

[G] C. McA. Gordon, Some embedding theorems and undecidability questions for groups, Combinatorial and geometric group theory (Edinburgh, 1993), 105-110, London Math. Soc. Lecture Note Ser., 204, Cambridge Univ. Press, Cambridge, 1995.

[K] R. Kirby, Markov's theorem on the nonrecognizability of 4-manifolds, Celebratio Mathematica, Volume on Martin Scharlemann, Mathematical Science Publishers, 2020.

[Mar] A.A. Markov, Insolubility of the problem of homeomorphy, Proc. Internat. Congress Math. 1958, pp 300-306, Cambridge Univ. Press, New York, 1960 .

[Mat] Ju.V. Matijasevič, Simple examples of unsolvable associate calculi, Dokl. Akad. Nauk SSSR 73 (1967), 1264-1266.

[Mi] C.F. Miller III, Decision problems for groups - survey and reflections, Algorithms and classification in combinatorial group theory, (Berkeley, CA, 1989), 1-59, Math. Sci. Res. Inst. Publ., 23, Springer, New York, 1992.

[N] P.S. Novikov, On the algorithmic unsolvability of the problem of identity, Trudy Mat. Inst. Steklov. no. 44. Izdat. Akad. Nauk SSSR, Moscow, 1955, $143 \mathrm{pp}$. 
[Ra] M.O. Rabin, Recursive unsolvability of group theoretic problems, Ann. of Math. 67 (1958), 172-194..

[Ru] J.H. Rubinstein, An algorithm to recognize the 3-sphere, Proceedings of the International Congress of Mathematicians, Vol. 1,2 (Zürich, 1994), 601-611, Birkhäuser, Basel, 1995.

[S1] M.A. Stan'ko, Markov's theorem and algorithmically non-recognizable combinatorial manifolds, Izv. Math. 68 (2004), 205-221.

[S2] M.A. Stan'ko, On the Markov theorem on algorithmic nonrecognizability of manifolds, J. Math. Sciences 146 (2007), 5622-5623.

[T] A. Thompson, Thin position and the recognition problem for $S^{3}$, Math. Res. Lett. 1 (1994), 613-630.

[VKT] I.A. Volodin, V.E Kuznetsov and A.T. Fomenko, The problem of the algorithmic discrimination of the standard three-dimensional sphere, Uspehi. Mat. Nauk 29 (1974), 71-168.

\footnotetext{
Department of Mathematics

University of Texas at Austin

1 University Station

Austin, TX 78712

USA.

gordon@math.utexas.edu
} 